\title{
On finite groups with dismantlable subgroup lattices
}

\author{
Marius Tărnăuceanu \\ February 17, 2015
}

\begin{abstract}
In this note we study the finite groups whose subgroup lattices are dismantlable.

MSC (2000) : Primary 20D30; Secondary 20D60, 20E15.

Key words : finite groups, subgroup lattices, dismantlable lattices, planar lattices, crowns.

\section{Introduction}

The relation between the structure of a group and the structure of its lattice of subgroups constitutes an important domain of research in group theory. The topic has enjoyed a rapid development starting with the first half of the 20th century. Many classes of groups determined by different properties of partially ordered subsets of their subgroups (especially lattices of (normal) subgroups) have been identified. We refer to Suzuki's book [9], Schmidt's book [7] or the more recent book [11] by the author for more information about this theory.

A finite lattice $L$ of $n$ elements is called dismantlable if there is a chain $L_{1} \subset L_{2} \subset \ldots \subset L_{n}=L$ of sublattices of $L$ such that $\left|L_{i}\right|=i$, for all $i=1,2, \ldots, n$ (see [5, 6]). It is well-known that every lattice with at most seven elements is dismantlable, while for every integer $n \geq 8$ there is a lattice of $n$ elements which is not dismantlable. Two basic properties of these lattices are the following:
\end{abstract}


- The class of dismantlable lattices is closed under the formation of sublattices and homomorphic images.

- If a dismantlable lattice is not a chain, then it contains at least two incomparable doubly irreducible elements.

Several characterizations of dismantlable lattices are known. One of the most significant is given by [5] and uses some particular partially ordered sets, namely crowns. Recall that for an integer $n \geq 3$, a crown of order $2 n$ is a poset $\left\{x_{1}, y_{1}, x_{2}, y_{2}, \ldots, x_{n}, y_{n}\right\}$ in which $x_{i} \leq y_{i}$ for all $i=1,2, \ldots, n$, $y_{i} \geq x_{i+1}$ for all $i=1,2, \ldots, n-1$, and $x_{1} \leq y_{n}$ are the only comparability relations.

Theorem 1.1. A finite lattice is dismantlable if and only if it contains no crown. In particular, a finite modular lattice is dismantlable if and only if it contains no crown of order 6 , or, equivalently, no sublattice isomorphic to the boolean lattice $\mathbf{2}^{3}$.

The connections between dismantlable lattices and planar lattices (i.e. lattices having a planar diagram) are powerful (see [1, 5]). In this way, every finite planar lattice is dismantlable. The converse is not necessary true, more precisely for every integer $n \geq 9$ there is an $n$-element dismantlable lattice which is not planar. However, for distributive lattices the above two concepts are equivalent.

Theorem 1.2. A finite distributive lattice is dismantlable if and only if it is planar.

Starting with the lattice-theoretical concept of "planar lattice", in [8] the classification of finite groups whose subgroup lattices are planar has been made, namely:

Theorem 1.3. A finite group $G$ has planar subgroup lattice if and only if it satisfies one of the following properties (where $p$ and $q$ are primes, $n \in \mathbb{N}_{0}$ and $m \in \mathbb{N})$ :

a) $G$ is cyclic of order $p^{n}$ or $p^{n} q^{m}$.

b) $G$ is a p-group lattice-isomorphic to $\mathbb{Z}_{p^{m}} \times \mathbb{Z}_{p}$.

c) $G$ is dihedral of order 8 or quaternion of order 8 or 16 . 
d) $G=P Q$, where $P \unlhd G,|P|=p, Q$ is cyclic of order $q^{m}$, and $\left|Q: C_{Q}(P)\right|=q$.

e) $G=P Q$, where $P \unlhd G,|P|=p^{2},|Q|=q$, and $Q$ operates irreducibly on $P$.

Inspired by the above classification and because dismantlable lattices and planar lattices are closely connected, the following question is very natural:

Which are the finite groups $G$ with dismantlable subgroup lattices?

This suggests us to consider the class $\mathcal{D}$ of groups satisfying the above property. Obviously, finite groups with at most seven subgroups are contained in $\mathcal{D}$, but at first sight it is difficult to determine all finite groups in $\mathcal{D}$. Therefore they must be investigated more carefully. Their study is the main goal of the current note.

Most of our notation is standard and will usually not be repeated here. Basic definitions and results on lattices and groups can be found in [2, 3] and [4, 10], respectively.

\section{Main results}

We start with the following easy but important lemma.

Lemma 2.1. Let $G$ be a finite group in $\mathcal{D}$. Then all elements of $G$ are of order $p^{n}$ or $p^{n} q^{m}$, where $p, q$ are primes and $n, m \in \mathbb{N}$.

Proof. Let $a \in G$. Then $L(\langle a\rangle)$ is a sublattice of $L(G)$ and consequently it is dismantlable. Since $L(\langle a\rangle)$ is also distributive (see e.g. Corollary 1.2.4 of [7]), we infer that it is planar by Theorem 1.2. Moreover, Theorem 1.3 shows that $o(a)=|\langle a\rangle|$ is of type $p^{n}$ or $p^{n} q^{m}$, as desired.

It is clear that the groups $A_{n}$ for $n \leq 4$ and $S_{n}$ for $n \leq 3$ belong to $\mathcal{D}$. On the other hand, we can easily check that the subgroups $H_{1}=\langle(12)(34)\rangle, K_{1}=$ $\langle(125),(12)(34)\rangle, H_{2}=\langle(25)(34)\rangle, K_{2}=\langle(143),(25)(34)\rangle, H_{3}=\langle(13)(25)\rangle$ and $K_{3}=\langle(15234),(13)(25)\rangle$ form a crown of order 6 in $A_{5}$, while the subgroups $H_{1}=\langle(12)\rangle, K_{1}=\langle(123),(12)\rangle, H_{2}=\langle(13)\rangle, K_{2}=\langle(134),(13)\rangle$, $H_{3}=\langle(14)\rangle$ and $K_{3}=\langle(124),(14)\rangle$ form a crown of order $6 \mathrm{in} S_{4}$. Consequently, $A_{5}$ and $S_{4}$ are not contained in $\mathcal{D}$. These remarks lead to the following result. 
Theorem 2.2. The alternating group $A_{n}$ is contained in $\mathcal{D}$ if and only if $n \leq 4$, while the symmetric group $S_{n}$ is contained in $\mathcal{D}$ if and only if $n \leq 3$.

Next we will focus on describing abelian groups $G$ contained in $\mathcal{D}$. Since the subgroup lattice of an abelian group is modular, Theorem 1.1 implies that $G$ belongs to $\mathcal{D}$ if and only if $L(G)$ does not contain sublattices isomorphic to the boolean lattice $\mathbf{2}^{3}$. We remark that for abelian $p$-groups this condition is equivalent with the fact that $G$ has no section of type $(p, p, p)$, i.e. it is of rank $\leq 2$.

Theorem 2.3. A finite abelian group $G$ belongs to $\mathcal{D}$ if and only if either it is cyclic and $|G| \in\left\{p^{n}, p^{n} q^{m}\right\}$, where $p, q$ are primes and $n, m \in \mathbb{N}$, or $G$ is a rank 2 abelian p-group.

Proof. The subgroup lattice of a cyclic group of order $p^{n}$ or $p^{n} q^{m}$ is planar by Theorem 1.3 and therefore such a group is contained in $\mathcal{D}$. On the other hand, we already have seen that rank 2 abelian $p$-groups belong to $\mathcal{D}$.

Conversely, assume that $G$ is contained in $\mathcal{D}$. Then Lemma 2.1 and the above remark show that either $G$ is an abelian $p$-group of rank $\leq 2$ or a direct product of two $p$-groups, say $G \cong G_{1} \times G_{2}$ with $\left|G_{1}\right|=p^{n}$ and $\left|G_{2}\right|=q^{m}$, $n, m \in \mathbb{N}^{*}$. In the second case if both $G_{1}$ and $G_{2}$ are cyclic, so is $G$ and we are done. Assume now that $G_{1}$ is not cyclic and take two maximal subgroups $M$ and $M^{\prime}$ of $G_{1}$. Then it is easy to see that the subgroups $H_{1}=M, K_{1}=G_{1}$, $H_{2}=M^{\prime}, K_{2}=M^{\prime} \times G_{2}, H_{3}=\left(M \cap M^{\prime}\right) \times G_{2}$ and $K_{3}=M \times G_{2}$ form a crown of order 6 of $L(G)$, a contradiction. This completes the proof.

An immediate consequence of Theorems 1.3 and 2.3 is the following.

Corollary 2.4. For every integer $n=p^{m}$, where $p$ is a prime and $m \geq 4$, there is a not-planar dismantlable lattice of $n$ elements which is isomorphic to the subgroup lattice of a certain finite abelian p-group.

We also observe that almost the same thing can be said about nilpotent groups contained in $\mathcal{D}$, namely:

Theorem 2.5. Let $G$ be a finite nilpotent group contained in $\mathcal{D}$. Then either $G$ is cyclic and $|G| \in\left\{p^{n}, p^{n} q^{m}\right\}$, where $p, q$ are primes and $n, m \in \mathbb{N}$, or $G$ is a non-cyclic p-group which does not contain abelian sections of type $(p, p, p)$. 
Unfortunately, we were not able to determine all non-cyclic $p$-groups with the above property. It is obvious that this is satisfied by rank 2 abelian $p$ groups, but several non-abelian $p$-groups also satisfy it, as shows the following theorem.

Theorem 2.6. All finite p-groups having a cyclic maximal subgroup are contained in $\mathcal{D}$. In particular, the non-abelian p-groups $M\left(p^{n}\right), D_{2^{n}}, Q_{2^{n}}$ for $n \geq 3$, and $Q D_{2^{n}}$ for $n \geq 4$ are contained in $\mathcal{D}$.

Proof. Let $n \geq 3$ be an integer and denore by $\mathcal{G}_{n}$ the class of $p$-groups of order $p^{n}$ possessing a cyclic maximal subgroup. This contain the cyclic $p$ group $\mathbb{Z}_{p^{n}}$, the abelian $p$-group $\mathbb{Z}_{p} \times \mathbb{Z}_{p^{n-1}}$, as well as the non-abelian $p$-groups described exhaustively in Theorem 4.1 of [10], II:

- the modular p-group

$$
M\left(p^{n}\right)=\left\langle x, y \mid x^{p^{n-1}}=y^{p}=1, y^{-1} x y=x^{p^{n-2}+1}\right\rangle,
$$

where $n \geq 4$ for $p=2$,

- the dihedral group

$$
D_{2^{n}}=\left\langle x, y \mid x^{2^{n-1}}=y^{2}=1, y x y=x^{-1}\right\rangle,
$$

- the generalized quaternion group

$$
Q_{2^{n}}=\left\langle x, y \mid x^{2^{n-1}}=y^{4}=1, y x y^{-1}=x^{2^{n-1}-1}\right\rangle,
$$

- the quasi-dihedral group

$$
Q D_{2^{n}}=\left\langle x, y \mid x^{2^{n-1}}=y^{2}=1, y x y=x^{2^{n-2}-1}\right\rangle,
$$

where $n \geq 4$.

Notice that for every non-cyclic $p$-group $G \in \mathcal{G}_{n}$ we have $|G: \Phi(G)|=p^{2}$. Moreover, all maximal subgroups of a group in $\mathcal{G}_{n}$ belong to $\mathcal{G}_{n-1}$.

We will prove by induction on $n$ that $\mathcal{G}_{n}$ is included in $\mathcal{D}$. Clearly, this holds for $n=3$. Assume now that $n$ is a minimal positive integer such that $\mathcal{G}_{n} \nsubseteq \mathcal{D}$ and take $G \in \mathcal{G}_{n} \backslash \mathcal{D}$. Let $\left\{H_{1}, K_{1}, H_{2}, K_{2}, \ldots, H_{m}, K_{m}\right\}$ be a crown of order $2 m$ in $L(G)$. By our assumption, we infer that there is no maximal subgroup $M$ of $G$ such that $K_{i} \subseteq M$ for all $i=1,2, \ldots, m$. Thus there exist 
at least two maximal subgroups $M_{1}$ and $M_{2}$ of $G$ containing subgroups in the set $\mathcal{K}=\left\{K_{1}, K_{2}, \ldots, K_{m}\right\}$. Since every $H_{i}$ is included in the intersection of two subgroups in $\mathcal{K}$, it follows that we can choose $H_{i_{1}} \neq H_{i_{2}}$ which are contained in $M_{1} \cap M_{2}=\Phi(G)$. In other words, the cyclic $p$-group $\Phi(G)$ contains two incomparable subgroups, a contradiction.

An important class of finite nilpotent groups is constituted by hamiltonian groups, that is non-abelian groups all of whose subgroups are normal. The structure of such a group $H$ is well-known, namely

$$
H \cong Q_{8} \times \mathbb{Z}_{2}^{n} \times A
$$

where $Q_{8}$ is the quaternion group, $n \in \mathbb{N}$ and $A$ is a finite abelian group of odd order. By Theorem 2.5 we easily infer that if $H$ belongs to $\mathcal{D}$, then $n=0$ and $A$ is trivial. In this way, a nice characterization of $Q_{8}$ is obtained.

Corollary 2.7. The quaternion $Q_{8}$ is the unique hamiltonian group contained in $\mathcal{D}$.

In d) and e) of Theorem 1.3 two types of semidirect products of orders $p q^{m}$ and $p^{2} q$, respectively, have been presented. These belong to $\mathcal{D}$ because their subgroup lattices are planar. We end our note by studying the containment to $\mathcal{D}$ for other remarkable semidirect products: dihedral groups (notice that these are not of type d) or e)). Recall that the dihedral group $D_{2 n}$ is the symmetry group of a regular polygon with $n$ sides and it has the order $2 n$. The most convenient abstract description of $D_{2 n}$ is obtained by using its generators: a rotation $x$ of order $n$ and a reflection $y$ of order 2. Under these notations, we have

$$
D_{2 n}=\left\langle x, y \mid x^{n}=y^{2}=1, y x y=x^{-1}\right\rangle .
$$

The subgroup structure of $D_{2 n}$ is precisely known: for every divisor $d$ or $n$, $D_{2 n}$ possesses a subgroup isomorphic to $\mathbb{Z}_{d}$, namely $\left\langle x^{\frac{n}{d}}\right\rangle$, and $\frac{n}{d}$ subgroups isomorphic to $D_{2 d}$, namely $\left\langle x^{\frac{n}{d}}, x^{i-1} y\right\rangle, i=1,2, \ldots, \frac{n}{d}$.

Theorem 2.8. The dihedral group $D_{2 n}$ is contained in $\mathcal{D}$ if and only if $n$ is of type $p^{m}$, where $p$ is a prime and $m \in \mathbb{N}$.

Proof. Assume first that $D_{2 n} \in \mathcal{D}$. By Lemma 2.1 we infer that $n=2^{k} p^{m}$ with $p$ an odd prime and $k, m \in \mathbb{N}$. If $m=0$, then $n$ is of the desired 
type. For $m \geq 1$ we will prove that $k=0$. Suppose $k \geq 1$. Then it is easy to see that the subgroups $H_{1}=\left\langle x^{2^{k-1} p^{m}}\right\rangle, K_{1}=\left\langle x^{2^{k-1} p^{m}}, y\right\rangle, H_{2}=\langle y\rangle$, $K_{2}=\left\langle x^{2^{k}}, y\right\rangle, H_{3}=\left\langle x^{2^{k}}\right\rangle$ and $K_{3}=\langle x\rangle$ form a crown of order 6 in $L\left(D_{2 n}\right)$, a contradiction.

Conversely, we already know that $D_{2^{m+1}}$ belongs to $\mathcal{D}$ by Theorem 2.6. Therefore we can assume that $p$ is odd. We will prove by induction on $m$ that $D_{2 p^{m}}$ is also contained in $\mathcal{D}$. This is obviously true for $m=1$ since $D_{2 p}$ and $\mathbb{Z}_{p} \times \mathbb{Z}_{p}$ are lattice-isomorphic. Let $m$ be minimal with the property $D_{2 p^{m}} \notin \mathcal{D}$ and take a crown of order $2 r$ in $L\left(D_{2 p^{m}}\right)$, say $\left\{H_{1}, K_{1}, H_{2}, K_{2}, \ldots, H_{r}, K_{r}\right\}$. We observe that the maximal subgroups of $D_{2 p^{m}}$ are $\langle x\rangle \cong \mathbb{Z}_{p^{m}}$ and $\left\langle x^{p}, x^{i-1} y\right\rangle \cong$ $D_{2 p^{m-1}}, i=1,2, \ldots, p$. Since by inductive hypothesis all $K_{i}$ 's cannot be contained in the same maximal subgroup of $D_{2 p^{m}}$ and by using a similar argument as in the proof of Theorem 2.6, one obtains that there are $H_{i} \neq H_{j}$ included in $\Phi\left(D_{2 p^{m}}\right)$. But $\Phi\left(D_{2 p^{m}}\right)=\left\langle x^{p}\right\rangle \cong \mathbb{Z}_{p^{m-1}}$ is cyclic, a contradiction. Hence $D_{2 p^{m}} \in \mathcal{D}$.

Finally, we indicate a natural open problem concerning the above study.

Open problem. Characterize arbitrary finite groups $G$ contained in $\mathcal{D}$. Is it true that all these groups are metacyclic?

Acknowledgments. We are grateful to Professor Roland Schmidt for his advices on the first version of our note.

\section{References}

[1] Baker, K.A., Fishburn, P.C., Roberts, F.S., Partial orders of dimension 2, interval orders, and interval graphs, Rand Corp. P-4376 (1970).

[2] Birkhoff, G., Lattice theory, Amer. Math. Soc., Providence, R.I., 1967.

[3] Grätzer, G., General lattice theory, Academic Press, New York, 1978.

[4] Isaacs, I.M., Finite group theory, Amer. Math. Soc., Providence, R.I., 2008.

[5] Kelly, D., Rival, I., Crowns, fences, and dismantlable lattices, Canad. J. Math. 26 (1974), 1257-1271. 
[6] Rival, I., Lattices with doubly irreducible elements, Canad. Math. Bull. 17 (1974), 91-95.

[7] Schmidt, R., Subgroup lattices of groups, de Gruyter Expositions in Mathematics 14, de Gruyter, Berlin, 1994.

[8] Schmidt, R., Planar subgroup lattices, Algebra Univers. 55 (2006), 3-12.

[9] Suzuki, M., Structure of a group and the structure of its lattice of subgroups, Ergebnisse der Mathematik und ihrer Grenzgebiete, Neue Folge, Heft 10, Springer-Verlag, Berlin-Göttingen-Heidelberg, 1956.

[10] Suzuki, M., Group theory, I, II, Springer Verlag, Berlin, 1982, 1986.

[11] Tărnăuceanu, M., Groups determined by posets of subgroups, Ed. Matrix Rom, Bucureşti, 2006.

\author{
Marius Tărnăuceanu \\ Faculty of Mathematics \\ "Al.I. Cuza" University \\ Iaşi, Romania \\ e-mail: tarnauc@uaic.ro
}

